\title{
Guest-Editorial
}

\section{Intelligent decision making in dynamic environments: methods, architectures and applications}

\author{
Alexander V. Smirnov ${ }^{\mathrm{a}}$ and Gabriel Jakobson ${ }^{\mathrm{b}}$ \\ ${ }^{a}$ St. Petersburg Institute for Informatics and Automation of Russian Academy of Sciences, Russia \\ E-mail: smir@mail.iias.spb.su \\ bAltusys Corp., USA \\ E-mail: jakobson@altusystems.com
}

This Special Issue presents recent research results in the fast growing area of intelligent decision technologies (IDT) in dynamic environments. Intelligent decision making in dynamic environments can be distinguished by a number of aspects from a conventional decision making. First, due to situational changes in an operational field, time is an inherent dimension of the decision making process, and as such it affects decision-making in several aspects, including speed, temporal relations between occurring events, and the order of the undertaken decision-making actions. Secondly, multiple alternative decision-making strategies can be applied depending on the assessment of current situations. Even more, the ongoing decisionmaking process may be dynamically changed, modified and optimized following unfolding external situations on the ground as well system internal resource situations. Thirdly, IDT processes in dynamic environments should maintain the truth of the logical decision making process and resolve conflicts. IDT in dynamic environments need construction and current updating of the complex situational picture, which might involve a large number of entities inter-related by structural, spatial, temporal, casual, and other domain-specific relations.

A variety of applications benefit from, and provide a sounding board for, technologies in dynamic environ- ments, including natural and human caused disasters management, tactical warfare, intelligent transportation systems, physical and cyber security management, and others. One of the major problems of intelligent decision making in such dynamic environments is the gap between the 'raw' sensor data and the information used by decision makers. The input to the information gathering process is hence defined by available sensors, and the desired output is defined by precondition constraints to standard operating procedures. The first paper of this issue is written by Gerhard Wickler and Stephen Potter, and it addresses the information-gathering process as a three-phase procedure that decomposes the overall problem into phases requiring different types of knowledge and information processing capabilities. The first phase, data validation, aims to remove incorrect information from the input data, thereby creating a consistent view of the current situation. The second phase, data abstraction and aggregation, applies mathematical models to reduce the amount of data, remove noise from the data, and derive features that are closer to the terminology of the user. The third phase, information interpretation, uses a belief revision and rulebased approach to make the information actionable for the decision maker.

One of the critical tasks of securing the effective and purposeful behaviour of large-scale distributed systems 
is coordination of activity of the system components. The second paper by Maurizio Marchese, Lorenzino Vaccari, Gaia Trecarichi, Nardine Osman, Fiona McNeill, and Paolo Besana addresses a novel approach to inter-component coordination based on shared protocol models that are distributed through a peer-to-peer infrastructure. The authors demonstrate how their proposed approach can be applied to support coalition formation and process coordination in in the context of crisis management.

The third paper in this issue belongs to Vladimir Gorodetsky, Oleg Karsaev, Vladimir Samoylov, Sergey Serebryakov describes the use of intelligent agent and the peer-to-peer (P2P) technologies to support the development of highly heterogeneous distributed decision making applications that are operating in a dynamic ubiquitous environment. The proposed technology is demonstrated by a case study of an air traffic control and emergency management in an airport area. This application is a typical example of a modern agentbased distributed decision making system, operating in a dynamic $\mathrm{P} 2 \mathrm{P}$ environment.
The next paper of the issue is written by Bradley Rhodes, Neil Bomberg, Majid Zandipour, Denis Garagic, Lauren Stolzar, James Dankert, Allen Waxman, and Michael Seibert. The paper discusses the algorithms of intelligent decision-making in dynamic environments that require learning of patterns of behavior in order to detect deviations from a normal behavior, and predict the future behavior. The authors present neurocognitively inspired learning algorithms and demonstrate the algorithms by applying them to the process of learning vessel behaviors across the maritime domain.

One of the most challenging tasks of intelligent decision-making in dynamic environments is situation awareness. Pradeep Ray and Subhagata Chattopadhyay in their paper describe how a. fuzzy awareness model can be used to analyze disaster situations, e.g. in pandemics that can be caused by the spread of diseases and which need effective treatment of the victims. The paper describes a cooperative intelligent information system for disaster situations that uses the method of fuzzy modeling for disaster situation awareness. 